Published by Al-Nahrain College of Medicine P-ISSN 1681-6579

E-ISSN 2224-4719

Email: iraqijms@colmed-alnahrain.edu.iq http://www.colmed-alnahrain.edu.iq http://www.iraqijms.net

\title{
Peroperative Factors which Achieve Successful Patellar Tracking in Primary Total Knee Replacement
}

\author{
Zaid A. Alshemmari FIBMS (Orth) \\ Dept. of Surgery, College of Medicine, Al-Nahrain University, Baghdad, Iraq
}

\begin{abstract}
Background Primary total knee arthroplasty (TKA) is technically demanding surgery, which if it is done perfectly can give excellent patient satisfaction and pain relief. Still abnormal patellar tracking is the most common complication of primary TKA (24\%) and it is the most common cause of revision.

Objective

To emphasize on the effectiveness of adherence to certain surgical steps (peroperatively) and to which extent these steps can reduce the incidence of peroperative abnormal patellar tracking in primary TKA.

Methods A prospective descriptive study was conducted in Al-Imamein Al-Kadhimein Medical City from May 2011 to December 2014; it involves 67 patients who underwent unilateral total knee replacement for different pathology and variable knee deformity. Step wise surgical procedure for primary TKA insisting on certain technique and tricks to achieve perfect TKA and finally checking the patellar tracking preoperatively using no thumb test and towel clips test.

Results NEXGENR posterior cruciate substitution design used in 62 patients (92.5\%) and condylar constrained knee in 5 patients (7.5\%) and abnormal patellar tracking occurred preoperatively only in 1 patient (1.4\%).

Conclusion Strict adherence to surgical procedures keeping in mind certain factors, which should be fulfilled is essential for successful peroperative patellar tracking, while postoperative assessment required different investigation like computed tomography or dynamic radiographs to ensure this good patellar tracking and improving the outcome of primary TKA in our country.

Keywords primary TKA, peroperative patellar tracking
\end{abstract}

DOI: $10.22578 / \mathrm{IJMS} .14 .4 .2$

List of abbreviation: TKA $=$ Total knee arthroplasty, PS = Posterior substitution, CCK = condylar constrained knee, LPS = Legacy posterior substitution

\section{Introduction}

$\mathrm{P}$ rimary total knee arthroplasty (TKA) is a technically challenging procedure that if performed with reasonable skill, can provide significant pain relief and patient satisfaction. The procedure is commonly done in most of specialized hospitals in Iraq in the last 4 years. The most frequent complications in primary total knee arthroplasty involve abnormal patellar tracking ${ }^{(1-6)}$, up to $24 \%$ and most common cause of revision $50 \%{ }^{(7)}$.
Optimizing patellar tracking in TKA requires attention to several peroperative factors, which include:

- Mechanical alignment of the femur and tibia.

- Femoral component rotation and sizing.

- Tibial component rotation.

- Femoral component coronal positioning.

- Tibial component coronal positioning.

- Patellar component positioning.

- Patellar height in Patellar resurfacing.

- Malposition of the tibial component in an internally rotated position increases the $Q$ angle by moving the tibial tubercle laterally. The increased $Q$ angle leads to lateral 
subluxation. The tibial component should be centered on the medial border of the tibial tubercle, with any deviation into slight external rotation. Similarly, internal rotation and medial translation of the femoral component move the trochlea more medial relative to the extensor mechanism, leading to lateral subluxation ${ }^{(3-5)}$.

The intraoperative evaluation of femoral component rotational alignment is based on anatomical landmarks. The posterior femoral condyles, epicondylar axis, and anteroposterior axis, all are useful in the primary knee arthroplasty setting. In revision arthroplasty, the position of the previous component and the epicondylar axis are usually the only landmarks available for this assessment. Malpositioned patellar, femoral, or tibial components also may lead to patellofemoral instability. Excessive lateral patellar facet resection is possible because of the normal asymmetry of the medial and lateral patellar facets. Often, the level of the lateral facet resection must be much shallower than the medial facet resection to avoid tilting of the patellar component. Lateral placement of the patellar component on the cut surface of the patella fails to reproduce the normal median eminence of the patella and can lead to lateral subluxation of the patella in extension. Studies by Hofmann et al and Lewonowski et al showed a reduction in lateral release rates in patients who had centralized patellar components.

By taking care of all these factors peroperatively and checking the patella tracking peroperatively by no thumb test (putting the knee through full range of motion with patella in its position without any pressure from the lateral side which should remain central with no tilt or shift) and reattachment of the patella to the medial retinaculum by towel clips and check patellar tracking, we can avoid any per operative lateral release of 7. patellar retinaculum and decrease the incidence of patellar necrosis ${ }^{(8)}$.

So the aim of this study was to assess the effectiveness of different surgical factors, which should be done perfectly during surgery to decrease the incidence of patellar maltracking which include (Mechanical alignment, femoral component rotation and sizing, tibial component rotation, femoral component coronal positioning, tibial component coronal positioning, Patellar component positioning and Patellar height in Patellar resurfacing.

\section{Methods}

This prospective study was conducted in Orthopedic Unit in Al-Imamein Al-kadimein Medical City from May 2011 to December 2014. It involved 67 patients who underwent unilateral total knee replacement. Their age were between 45 years and 70 years $(60$ female and 7 male) (Figure 1). The indications for TKA were osteoarthritis in 62 patient and rheumatoid arthritis in 5 patients (Figure 2).

All these knees replaced using the NEXGENR posterior cruciate substitution (PS) high flexed design and condylar constrained knee (CCK) system of the prosthesis.

\section{Inclusion criteria}

1. Male or female patient aged between $35-80$ years.

2. Patients with osteoarthritis and rheumatoid arthritis.

3. Varus deformity less and more than 15 degree.

4. Valgus deformity less and more than $\mathbf{1 5}$ degree.

5. Patients with range of motion more than 80 degrees with or without flexion contracture.

6. All patients have their first total knee replacement. 


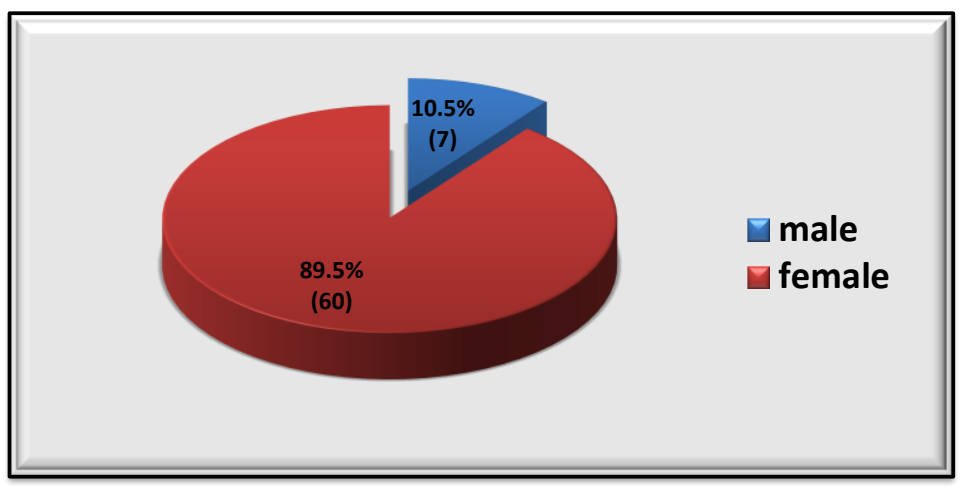

Figure 1. Pie chart shows distribution of the sample according to the gender

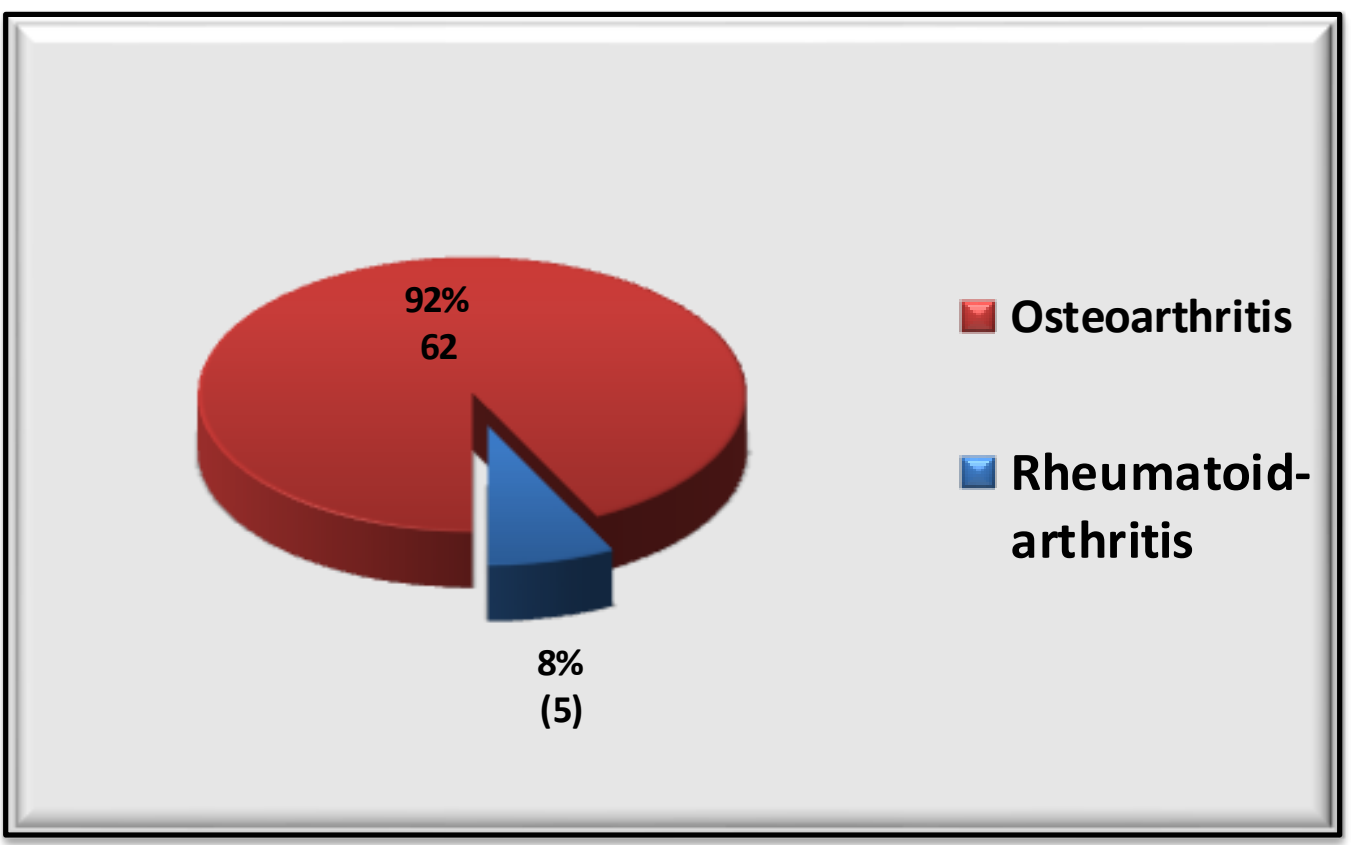

Figure 2. Pie chart chows distribution of the sample according to the underling pathology

\section{Exclusion criteria}

1. Patients with previous knee infection.

2. Patients with previous knee trauma or osteotomy around the knee or bony defect more than $5 \mathrm{~cm}$.

3. Previous patellar fracture.

4. Patients with range of motion less than 80 degrees.

5. Patients with neuromuscular or sever traumatic knee instability.

6. Any patient with revision.
Osteoarthritis is the main indication in 62 patients (92\%), and rheumatoid arthritis in 5 patient (8\%).

The left knee replaced in 35 patient (52\%) and 32 (48\%) right knee (Figure 3 ).

The deformity was varus in 58 (86.5\%), 12 of them had sever deformity (more than 15 degree), and valgus in 8 (11.9\%), 3 of them had severe deformity (more than 15 degree) and recarvatum in one patient (1.5\%). Six patients 
had flexion contracture more than 30 degree all are with sever varus deformity, and the others were easy primary total knee replacement (varus and valgus deformity less than 15 degree, range of motion more than 110, flexion contracture less than 15 degrees, no infection or trauma or knee instability or previous osteotomy (Figure 4) (Table 1).

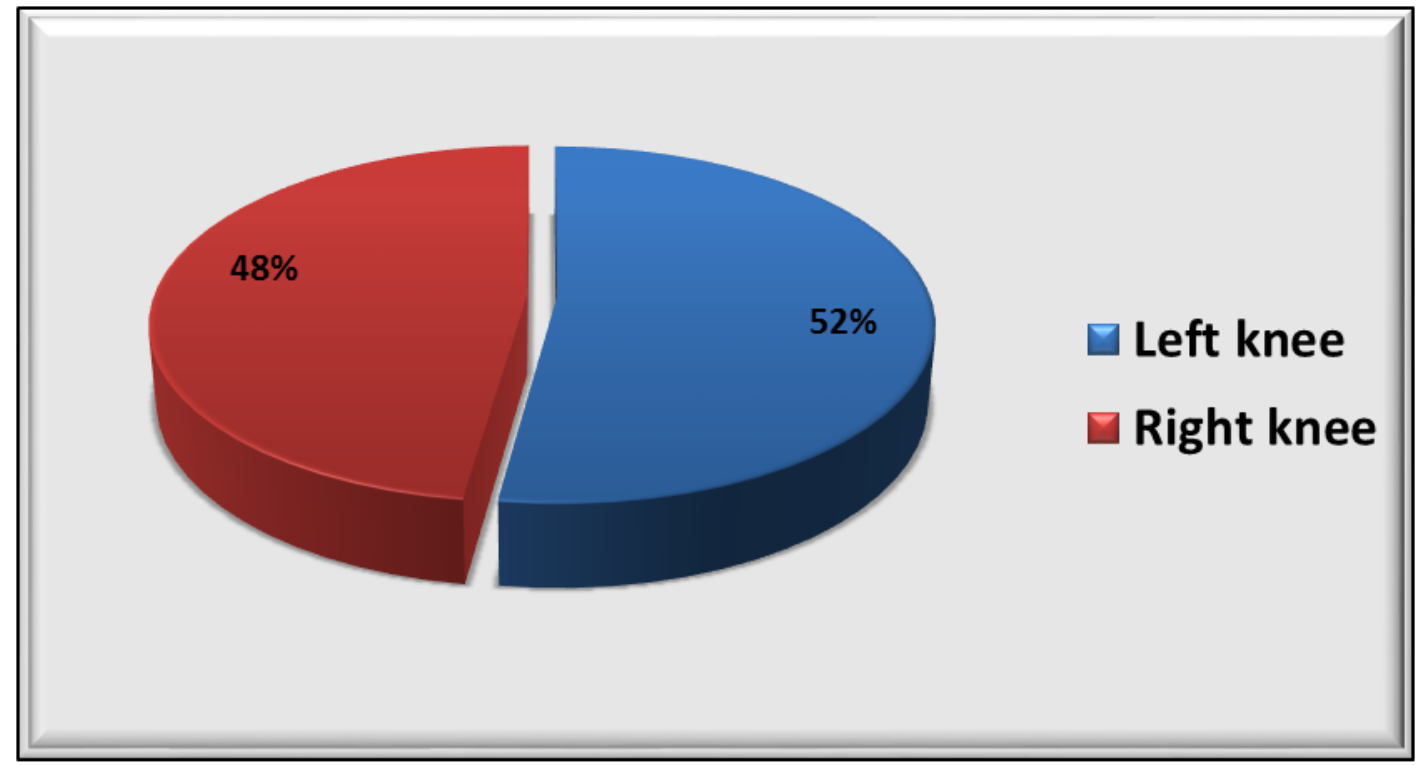

Figure 3. Pie chart shows distribution of sample according to the side affected

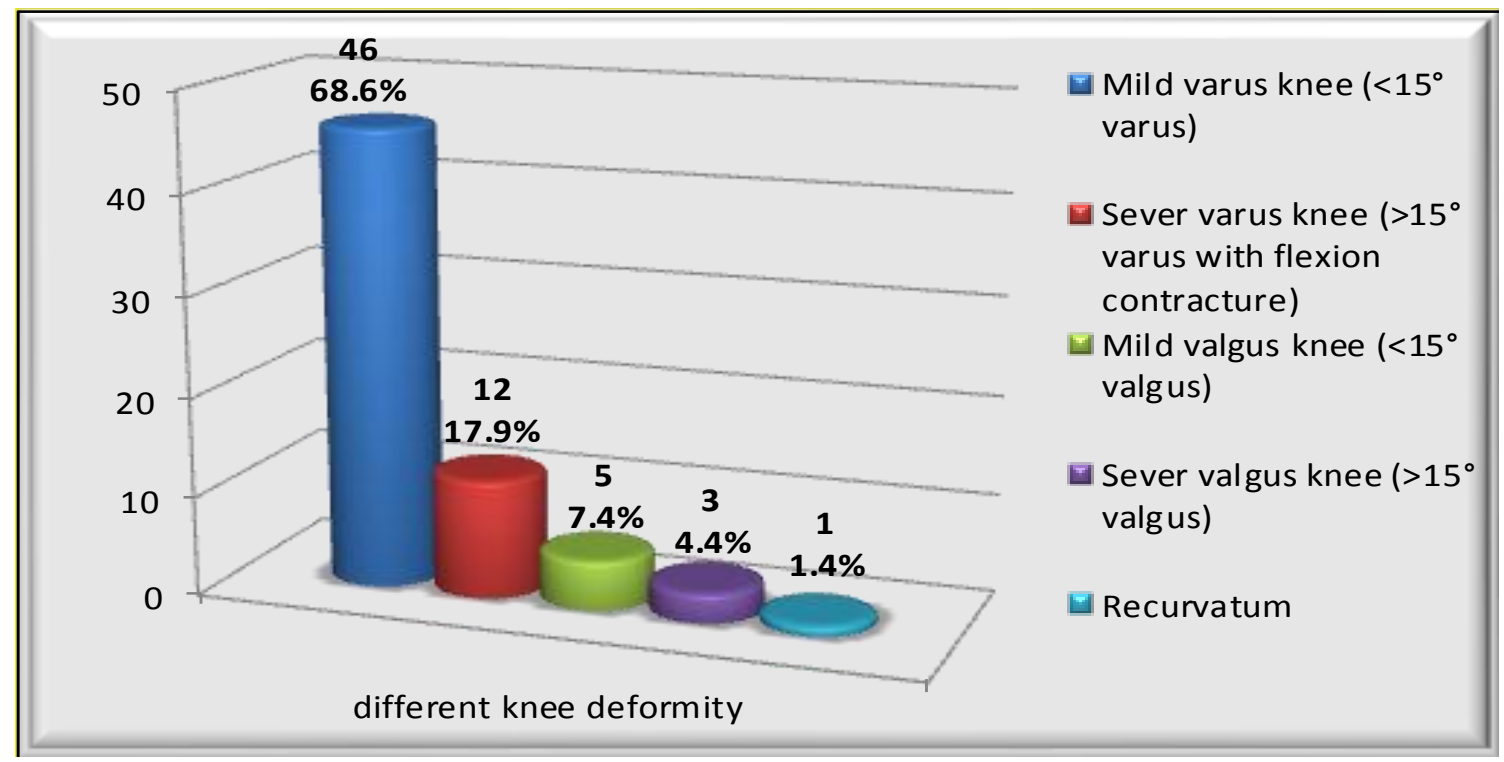

Figure 4. Bar chart show distribution of patients according different knee deformity 
Table 1. Frequency distribution of the patients according to the different knee deformity ( $n=67)$

\begin{tabular}{lcc}
\hline \multirow{2}{*}{ Different knee deformity } & \multicolumn{2}{c}{ Patients } \\
\hline Mild varus knee $\left(<15^{\circ}\right.$ varus $)$ & NO. & $\%$ \\
Sever varus knee $\left(>15^{\circ}\right.$ varus with flexion contracture) & 46 & 68.6 \\
Mild valgus knee $\left(<15^{\circ}\right.$ valgus) & 12 & 17.9 \\
Sever valgus knee $\left(>15^{\circ}\right.$ valgus) & 5 & 7.4 \\
Recurvatum & 3 & 4.3 \\
\hline Total & 1 & 1.4 \\
\hline
\end{tabular}

Legacy posterior substitution (LPS) posterior cruciate substitution was used in 62 (92.5\%) and CCK condylar constrained knee in 5 (7.5\%) cruciate retaining was not used in any patient. All the patients prepared well preoperatively regarding medical fitness and proper examination of the limb and the knee.

Long leg standing radiograph of lower limb was done with measurement of anatomical mechanical axis, lateral distal femoral axis, medial proximal tibial angle; varus or valgus angle.

All patients approached through anteromedial line skin incision and medial parapatellar incision. With the use of leg holder, and the use of pneumatic tourniquet is optional during the procedure.

\section{Surgical procedure}

1. proper distal femoral and proximal tibial bone cut fallowed by checking the mechanical axis of the cut from the femoral head to the center of ankle using special device passing through knee spacer.

2. Accurate femoral sizing using special jig provided by the set depending on the superolateral portion of femoral condyle, and lateralization of four in one cutting block.

3. Accurate external rotation of femoral component depending on the epicondylar axis mainly and to lesser extent the whitesite line and post condylar axis. IN most of our patient femoral component was implanted in 3 degree external rotation and few in 5 degree non in 0 degree.

4. Accurate tibial tray implantation by accurate rotation through putting the center of the tibial tray with the junction of lateral $2 / 3$ and med $1 / 3$, and accurate sizing by putting the curve of the tray on the ant curve of the tibial cut and avoiding medial and lateral displacement or over hang.

5. Only 3 patients in our series had patellal resurfacing (two of them with rheumatoid arthritis and one with sever patellar damage and abnormal shape by long standing history of osteoarthritis), in the others patellar denervation by cauterization of surrounding tissue up to $4 \mathrm{~mm}$ depth and denution of abnormal osteophyte was done.

6. In between the steps repeated trial to check each step was done using different trial devices.

7. Multiple checks of the patellar tracking by insuring that the patellar tendon covering the middle of tibial tuberosity and the middle of tibial tray.

8. Finally patellar tracking in trial run and after implantation of final implant is checked by NO THUMB TEST \{the position of patella is checked through full range of motion from full extension to full flexion with the patella in its position in front of knee and no any pressure applied to lateral side of the patella by thumb the patella should track smoothly with no any lateral shift or subluxation or dislocation or tilt with or without reattachment of the patella to 
medial retinaculum by towel clips $\}$ and rechecking of this tracking after towel clips application.

9. Proper closure of the arthrotomy and skin over two drains.

10. Final checking of range of motion, stability, alignment, and patellar tracking is done in every case.

\section{Results}

LPS posterior cruciate substitution was used in $62(92.5 \%)$ and CCK condylar constrained knee in $5(7.5 \%)$, while cruciate retaining was not used in any patient (Figure 5) (Table 2).

Table 2. Frequency distribution of the patients according to the types of knee prosthesis $(n=67)$

\begin{tabular}{lcc}
\hline \multirow{2}{*}{ Different knee deformity } & \multicolumn{2}{c}{ Patients } \\
& No. & $\%$ \\
\hline LPS & 62 & 92.6 \\
CCK & 5 & 7.4 \\
\hline Total & 67 & 100 \\
\hline
\end{tabular}

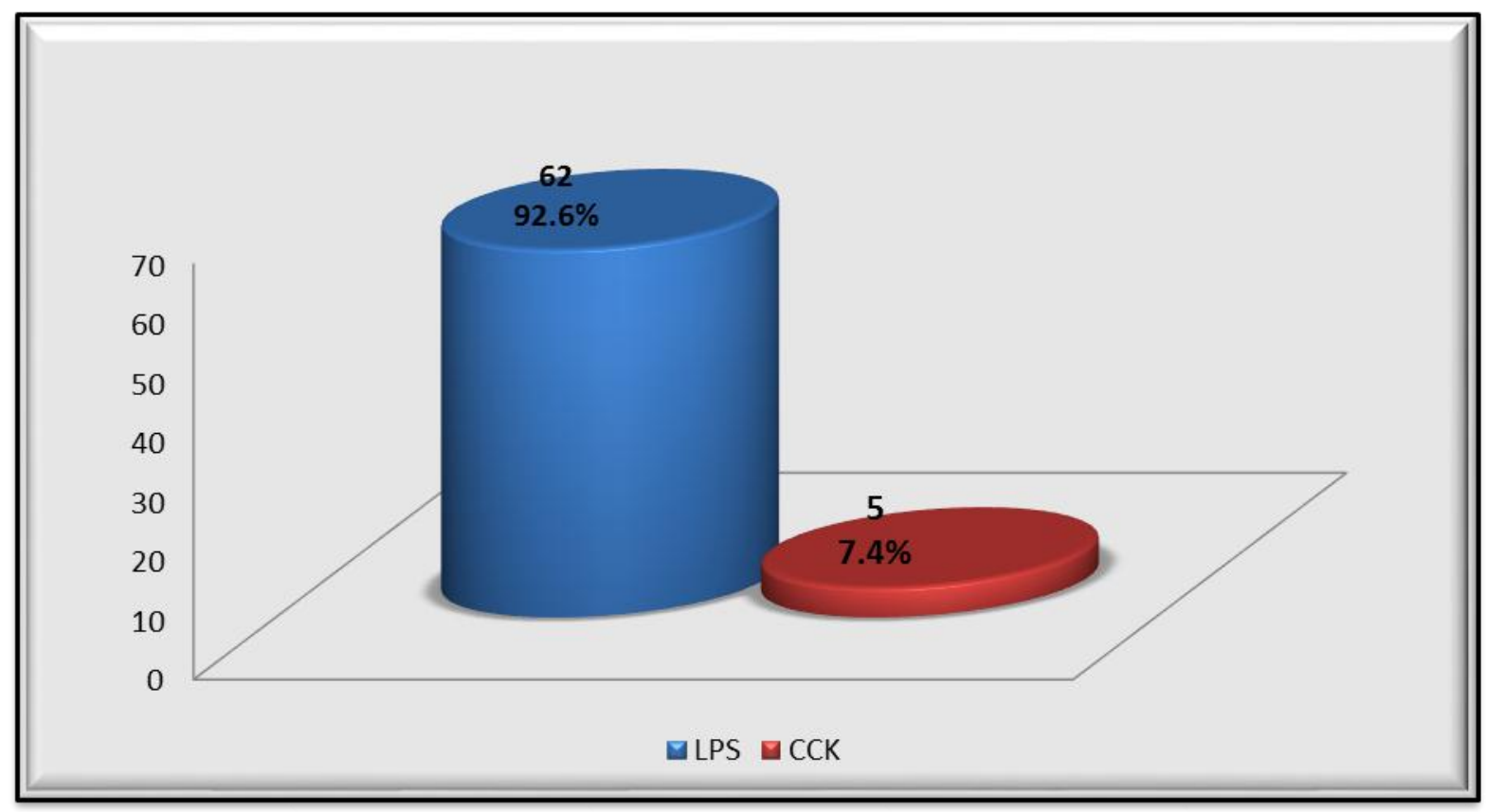

Figure 5. Bar chart shows distribution of patients according to the types of knee prostheses

CCK was used for the following conditions: 2 cases due to severe flexion extension gap mismatch, 2 cases due to sever varus deformity and incompetence of the collateral ligament, 1 case due to recarvatum and severe valgus deformity.

Fallowing the strict surgical steps during each operation of primary TKA result in: abnormal patellar tracking occur in 1 patient (1.4\%), which has recurvatum knee and required lateral release, and in 66 patient $(98.6 \%)$ with different types of prosthesis and different types and degrees of deformity have no abnormal patellar tracking peroperatively as checked by no thumb test and towel clips test (Figure 6). Discussion

The patella with or without replacement plays essential role in success of TKA and most of patellofemoral complications related to the 
patient, surgical technique or the design of the component ${ }^{(9)}$.

Patellar instability after TKA is serious complication, its etiology can be related to the surgical technique and component position, extensor mechanism imbalance ${ }^{(10)}$, Which was insisted on in this study.

The presence of anterior knee pain, especially during stressful activity is indication of patellar instability ${ }^{(10)}$.

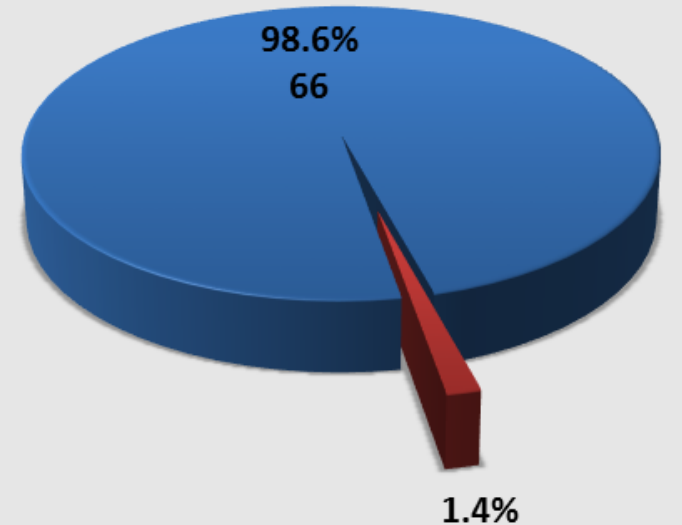

(1)

Figure 6. Pie chart shows distribution of the sample according to the patellar tracking

Assessing intraoperative tracking during TKA is important to avoid postoperative patellar complication, no thumb test, the towel clips test and vertical patellar test, which is novel technique to assess lateral retinaculum release (11), the use of no thumb test and reevaluation with towel clips test may reduce unnecessary lateral retinaculum release ${ }^{(12)}$ and so in this study no thumb test and towel clips test were used as the most dependable peroperative tests to assess patellar tracking.

Patellofemoral instability result from component malposition and limb malalignment, prosthetic design, improper patellar preparation and soft tissue imbalance; nonsurgical treatment is generally unsuccessful (13).

Although patellofemoral radiograph may display lateral subluxation, only computed tomography can quantify rotational malalignment of femoral and tibial components ${ }^{(13)}$.

Patellar instability may occur after TKA with or without patellar resurfacing, subluxation is more common than dislocation the incidence of symptomatic instability leading to revision is $(0.5 \%-0.8 \%)^{(14,15)}$. In this study, the incidence of patellar instability is $(1.4 \%)$, the case is recarvatum knee complicated by negative proximal tibial slope which lead to abnormal extensor mechanism.

Anglin et al (16) stated that the major determinant of patellar tilt and shift were patellar component medicalization, patellar resection angle and femoral component rotation, neither tibial component rotation nor patellar thickness should be adjusted to improve patellar tracking ${ }^{(16)}$. However, it was insisted that accurate positioning of all component, mechanical limb alignment accurate bone cuts, and soft tissue balancing 
lead to decrease abnormal patellar tracking in TKA and reduce the postoperative knee pain and the need for revision.

This study concluded that strict adherence to every step in the surgical procedure and accepting nothing less than perfect in every step whether on the femur or tibia or patella and whole alignment and soft tissue balancing, can achieve perfect patellar tracking peroperatively and reduce the need of lateral retinaculum release, which by itself increase post-operative complication.

This study recommend further studies to assess the importance of this perfect patellar tracking during surgery and its effectiveness in reducing the postoperative patellofemoral complication and by then reducing the incidence of revision. Also it is recommended that next studies fallow the patients for several months to assess how peroperative patellar tracking affect postoperative patellofemoral and knee function clinically and with the use of post-operative knee computed tomography scan and special dynamic radiological views to assess patellar tracking.

\section{Acknowledgments}

A great gratitude to Professor Dr. Abd-Ali Muhsin for his support. Sincere thanks to Assist. Professor Dr. Ahmed Sabeeh for his great help in doing many of these surgeries and directing the research. Great thanks to the theater staff in Al-Imamein Al-Kadhimein Medical City and all our patients.

\section{Conflict of interest}

The author declares no conflict of interest.

\section{Funding}

The operations and the follow up were done in Al-Imamein Al-Kadhimein Medical City, which is a governmental hospital, the theatre, surgical sets, instruments and the knee prosthesis provided by the hospital free of charge.

\section{References}

1. Parker D, Dunbar $M$, Rorabeck C. Extensor mechanism failure associated with total knee arthroplasty: prevention and management. J Am Acad Orthop Surg. 2003; 11(4): 238-47.

2. Ritter $M$, Herbst $S$, Keating $E$, et al. Patellofemoral complications following total knee arthroplasty. Effect of a lateral release and sacrifice of the Superior lateral geniculate artery. J Arthroplasty. 1996; 11(4): 368-72.

3. Theiss SM, Kitziger KJ, Lotke PS, et al. Component design affecting patellofemoral complications after total knee arthroplasty. Clin Orthop. 1996; 326: 1837.

4. Leblanc J. Patellar complications in total knee arthroplasty. A literature review. Orthop Rev. 1989; 18(3): 296-304.

5. Brick G, Scott R. The patellofemoral component of total knee arthroplasty. Clin Orthop. 1988; 231: 16378.

6. Mont $\mathrm{M}$, Yoon $\mathrm{T}$, Krackow $\mathrm{K}$, et al. Eliminating patellofemoral complications in total knee arthroplasty: clinical and radiographic results of 121 consecutive cases using the Duracon system. J Arthroplasty. 1999; 14(4): 446- 55.

7. Rand JA. The patellofemoral joint in total knee arthroplasty. J Bone Joint Surg Am. 1994; 76(4): 61220.

8. Canale ST, Beaty JH. Campbell's operative orthopedics. 11th ed. Philadelphia: Mosby; 2007. Chapter 6.

9. Gasparini G, Familiari F, Ranuccio F. Patellar malalignment treatment in total knee arthroplasty. JTS Joints CIC Edizioni Internazionali 2013; 1(1): 1017.

10. Motsis EK, Paschos N. Patellar instability after TKA. J Orthop Surg. 2009; 17(3): 351-7.

11. Goyal N, Mater WY, Parvisi J. Assessing patellar tracking during TKA: A technical note. Am J Orthop. (Belle Mead NJ). 2012; 41(10): 450-1.

12. Cho WS, Woo JH, Park HY, et al. Should the 'no thumb technique' be the golden standard for evaluating patellar tracking in total knee arthroplasty? The Knee. 2010; 18(3): 177-9.

13. Malo $M$, Vince KG. The unstable patella after TKA, etiology, prevention, management. J Am Acad Orthop Surg. 2003; 11(5): 364-71.

14. Rand JA. The patellofemoral joint in TKA. J Bone Joint Am. 1994; 76: 612-20.

15. Scuderi GR, Insal JN, Scott NW. Patellofemoral pain after TKA, JAM Acad Orthopedic Surg. 1994; 2: 23446.

16. Anglin C, Brimacombe JM, Hodgson AJ, e al. Determinants of patellar tracking in total knee arthroplasty. Clin Biomech (Bristol, Avon). 2008; 23(7): 900-10.

E-mail: zaidortho@yahoo.com Received $4^{\text {th }}$ Apr. 2016: Accepted $16^{\text {th }}$ Nov. 2016 